\title{
Preliminary Analyses of Controlled Release of Potassium Permanganate Encapsulated in Polycaprolactone
}

\author{
Niya S. King1, Stephanie Luster-Teasley², Clayton J. Clark II ${ }^{{ }^{*}}$ \\ ${ }^{1}$ Department of Civil and Environmental Engineering, Florida A \& M University, FAMU-FSU College of Engineering, \\ Tallahassee, FL, USA \\ ${ }^{2}$ Department of Civil, Architectural and Environmental Engineering, North Carolina A \& T State University, Greensboro, NC, USA \\ Email: *clark@eng.famu.fsu.edu,clayton.clarkii@famu.edu
}

How to cite this paper: King, N.S., Luster-Teasley, S. and Clark II, C.J. (2021) Preliminary Analyses of Controlled Release of Potassium Permanganate Encapsulated in Polycaprolactone. Journal of Water Resource and Protection, 13, 32-43.

https://doi.org/10.4236/jwarp.2021.131003

Received: December 19, 2020

Accepted: January 25, 2021

Published: January 28, 2021

Copyright $\odot 2021$ by author(s) and Scientific Research Publishing Inc. This work is licensed under the Creative Commons Attribution International License (CC BY 4.0).

http://creativecommons.org/licenses/by/4.0/

\begin{abstract}
Potassium permanganate $\left(\mathrm{KMnO}_{4}\right)$ has been used widely as an oxidant for remediation of contaminated soil and water systems. The present study evaluates the release of this oxidant from Polycaprolactone (PCL) polymer as part of a patented controlled release process (CRP) to be applied for targeted removal of contaminants from water. $\mathrm{KMnO}_{4}$ was encapsulated into PCL at a 1:5 oxidant to polymer ratio and placed in batch reactor systems with reagent water to be evaluated over a 96 hour period. SEM images showed that over time, the number of cavities and their sizes increased on the waxy surface of the PCL polymer. The experimental data from the release of $\mathrm{KMnO}_{4}$ from PCL was found to fit non-Fickian diffusion model after dissolution $\left(\mathrm{R}^{2}=\right.$ 0.93) similar to other systems that describe the dispersal of other oxidants from wax matrices. In addition, the model parameters for data of this present study were also found to be comparable to previous release studies with the same oxidant encapsulated in different wax matrices at similar ratios. Overall, the similarity of release data between the diversity of polymers shows that the controlled release biodegradable polymer utilizing PCL provides effective release of the $\mathrm{KMnO}_{4}$ with the added benefit biodegradable nature of PCL.
\end{abstract}

\section{Keywords}

Potassium Permanganate, Controlled-Release, Encapsulation, Kinetics

\section{Introduction}

Remediation of water and soil systems has long utilized various oxidation methods to treat contaminants in the environment. The aggressiveness of most 
oxidants to oxidize all organic materials has led to encapsulation of these chemicals in various polymers to cause their slow controlled release in these aqueous and soil systems [1] [2] [3]. In Situ Chemical Oxidation (ISCO) is the process that introduces the selected oxidant into the contaminated environment to reduce the harmful effects on the media [4] [5] [6] [7]. To increase efficiency of targeted oxidant contact in ISCO processes, Controlled Release Material (CRM) delivery systems have been developed for environmental purposes such as environmental protection, decontamination, and remediation, especially during treatment of groundwater, wastewater, and drinking water [8] [9]. Throughout the evolution of CRM, synthetics have been utilized to deliver the chemical oxidants including polyester mesh bags, Polymethyl Methacrylate (PMMA), Piccolyte resin S115, Epolene C-16, and stearic acid [10] [11] [12] [13]. However, often these synthetic polymers did not have a high rate of biodegradability and demonstrated harm to the environment and its organisms [14]. In response to these deficiencies, several studies have reported the safe and successful controlled slow-release of chemical oxidants using clay, waxes, and gels for remediation in various water systems [1] [2] [15]. However, the work to find the optimum oxidant for these controlled slow-release systems continues, as system and contaminant variability is challenging.

Various oxidants have been used for subsurface remediation such as hydrogen peroxide, persulfate, Fenton's reagent, and zero-valent iron [16] [17] [18] [19]. One highly effective oxidizing chemical agent for the removal of contaminants and the inactivation of microorganisms has been potassium permanganate [10] [20] [21]. In numerous instances, potassium permanganate $\left(\mathrm{KMnO}_{4}\right)$ has been found to produce environmentally harmless by-products in the degradation of organic and inorganic compounds within soil and groundwater [13] [22] [23] [24]. Other overall advantages of utilizing $\mathrm{KMnO}_{4}$ in remediation include its chemical stability, cost effectiveness, and the less hazardous biodegradable composition of the oxidant [25] [26]. Yet, the level of effectiveness of the $\mathrm{KMnO}_{4}$ for remediation is in direct correlation to the method its delivery. Methods for $\mathrm{KMnO}_{4}$ delivery have included pressurized direct-push, soil mixing, well flushing, treatment walls and oxidant, however these did not offer sufficient delivery control of the chemical oxidant [13]. This uncontrolled flow of the oxidant through these non-restrictive processes resulted in reactions with natural organic matter and inorganic soil constituents, such as metals. This reduced the amount of oxidant attacking the target contaminant and would often require additional measures to further remediate the media [1] [2]. Controlled-release delivery methods have been applied to $\mathrm{KMnO}_{4}$ with some success [7] [27] [28] [29]. Despite these applications, there is still much more information to be gathered surrounding polymers and their potential to increase delivery efficiency while reducing the negative impacts and by-products.

One polymer that has shown great promise regarding oxidant encapsulation for remediation of water sources is Polycaprolactone (PCL). PCL has a relatively 


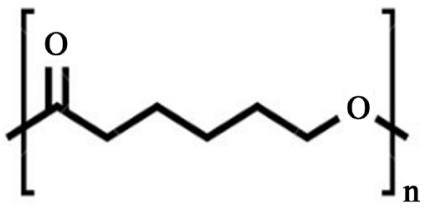

Figure 1. Structure of Polycaprolactone (PCL).

long half-life with little or no residual side effects or change in the molecular weight of the internal bulk of the polymer [14]. Bulk degradation of the PCL transpires when hydrolysis occurs after the entire polymer has been penetrated with water. In addition, PCL has a low melting point, exceptional blending capabilities, malleability into large ranges of shapes and sizes, and has been FDA approved [14] [30]. The long chain structure of PCL, which includes five non-polar methylene groups and a polar ester group (Figure 1), taken into account with its physiochemical characteristics make PCL an excellent candidate for CRM in water treatment systems [31].

This attractiveness of PCL as a CRM has led to it being central to a patented process (US Patent 8,519,061 B2) for controlled release of $\mathrm{KMnO}_{4}$ [32]. The patent entitled Controlled Released Biodegradable Polymer (CRBP) utilizes PCL for controlled delivery of the chemical oxidant to treat contaminated water systems over a particular span of time which may reduce the number of times the treatment needs to be administered [6] [21] [24]. Though these studies show promise, more information on the specifics of the oxidant release with respect to aqueous systems I s necessary. Once the release of the oxidant from PCL is better understood, the CRBP mechanism would be of more robust use in the oxidation of organic contaminants in soil and water. The main objective of the present study is to evaluate the release characteristics of $\mathrm{KMnO}_{4}$ encapsulated in PCL in an aqueous system.

\section{Materials and Methods}

\subsection{Materials}

Potassium permanganate, $\mathrm{KMnO}_{4}$, (ACS reagent grade, 99\%) was purchased as dark purple odorless crystals from Sigma Aldrich. The hydrophobic biodegradable polymer, Polycaprolactone, was also purchased from Sigma Aldrich. All solutions used in this study were prepared using ultrapure water from a bench ultrapure water system. As a biocompatible and biodegradable polymer, PCL was used to encapsulate $\mathrm{KMnO}_{4}$ to formulate a CRBP system to observe controlled-release kinetics and characteristics of encapsulated $\mathrm{KMnO}_{4}$ when introduced to aqueous media.

\subsection{Encapsulation of $\mathrm{KMnO}_{4} \mathrm{CRBP}$}

In this study, the first phase consisted of formulating CRBP pellets using the baking method for encapsulation described by the literature [32]. To produce the CRBP delivery system, $\mathrm{KMnO}_{4}$, as the chemical oxidant, and PCL, as the hy- 
drophobic polymer, were utilized. The encapsulation of this $\mathrm{KMnO}_{4}$ oxidant allowed for stabilizing control of chemical release. The study modeled basic reproducible components of Teasley et al. studies [6] [21] [24] for establishment of standard measurements including a $\mathrm{KMnO}_{4}$ to PCL at a ratio of $1: 5$ or $20 \mathrm{wt} \%$. The same standard measurements were used for all CRBP pellet formulations. Initially, using a laboratory muffle furnace (Thermo Scientific, US), PCL was heated at $90^{\circ} \mathrm{C}$. Once PCL was molten, $\mathrm{KMnO}_{4}$ particles were added to the soften polymer and stirred thoroughly to achieve a homogeneous mixture using a stainless-steel spatula. After blending for uniform particle dispersion, and while malleable, the molten mixture was then placed in a Parr stainless steel pellet press and pelletized. Solid circular pellet structures were produced with $20 \mathrm{wt} \%$ $\mathrm{KMnO}_{4}-\mathrm{PCL}$ and size of $1.3 \mathrm{~cm}$ in length capable of the controlled-release (Figure 2(a)). Pellets were then preserved and stored in a dry cool place until use for batch release experiments. Pellets were designed based on a combined mechanism [32] consisting of controlled dissolution and diffusion of $\mathrm{KMnO}_{4}$ for an extended period of time.

\subsection{Release Experiments}

The study was conducted in the form of a series of batch release experiments, similar to what is seen in Figure 2(b). Experiments were conducted with 1:5 ratio of CRPB $\mathrm{KMnO}_{4} / \mathrm{PCL}$ (also referred to as $20 \mathrm{wt} \%$ ), approximately $0.60 \mathrm{~g}$ in mass, pellets submerged in $1 \mathrm{~L}$ of ultra pure water and synchronously released over the 96 hour period. Each of 12 Pyrex glass beakers were used as batch reactors for individual pellets. These reactors were tightly capped at room temperature under constant mixing with a mechanical stirrer to ensure a homogenous mixed solution. Aluminum foil was wrapped around the glass reactors for protection from ambient light. Preliminary experiments confirmed the findings of prior studies noted that the water volume did not affect the amount of relative mass lost over a $24 \mathrm{~h}$ period [7]. The release of $\mathrm{KMnO}_{4}$ from the PCL was observed periodically by measuring the oxidant concentration in the aqueous solution. Samples of aqueous solution were withdrawn using a sterile serological pipette at various times within the 1 -week period. At each time interval after

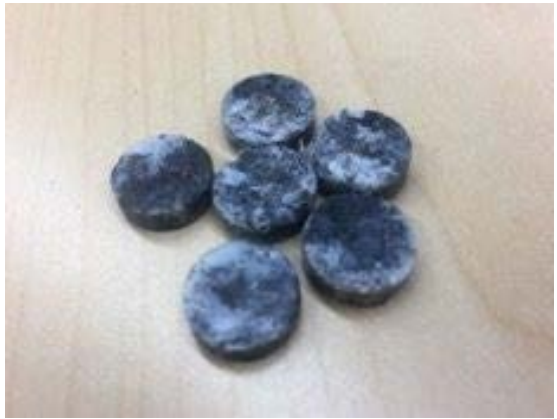

(a)

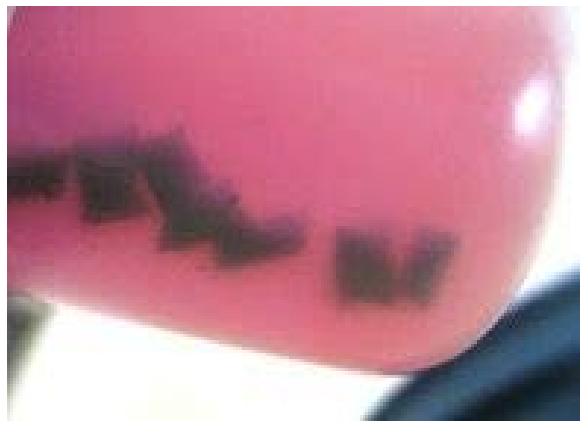

(b)

Figure 2. (a) Pellets with $\mathrm{KMnO}_{4}$ encapsulated in PCL; (b) Pellets release $\mathrm{KMnO}_{4}$ in water. 
release, pellets were collected from reactors, desiccated for 24 hours and weighed for calculating changes and averages in mass.

\subsection{Analysis}

Water samples collected from the batch experiments were analyzed for $\mathrm{KMnO}_{4}$ concentrations using a UV-Vis spectrophotometric by measuring the absorption at $\lambda=525 \mathrm{~nm}$ wavelength at each sampling time interval, which is within the maximum absorbance range for $\mathrm{KMnO}_{4}$. UV-Vis spectrophotometric analysis indicated the amount of additive $\mathrm{KMnO}_{4}$ integrated in the aqueous solution. $\mathrm{UV}$-Vis spectrophotometric used the absorbance measurements to determine the levels of $\mathrm{KMnO}_{4}$ concentration in the aqueous solution. The analyzed absorbance and concentration for the samples were taken for each interval of time. The surface morphology of the encapsulated $\mathrm{KMnO}_{4} \mathrm{CRBP}$ before and after release experiments were characterized using FEI Helious G4 UC field emission scanning electron microscope (FESEM).

\section{Results and Discussion}

The $\mathrm{KMnO}_{4}$ oxidant encapsulated in PCL polymer was released in reagent grade water to measure release characteristics of the system. Throughout the experimentation, the fraction of the oxidant released was seen to continually increase over the 96 hour sampling period for the present study (Figure 3). As with similar experiments, the greatest percentage of oxidant release was within the first 24 hours, reaching the maximum rate of release between the 12 hour and 24 hour time interval. This correlates to the prior findings related to this rapid release biphasic phase in the CR process [2] [33].

\subsection{Surface Morphology of the CRBP}

The release of the $\mathrm{KMnO}_{4}$ particles from the PCL matrix can readily be seen in the SEM images (Figure 3). Increasing oxidant removal from the PCL surface was evidenced by the increase in size of cavities in the waxy surface of the polymer as time from release increased. These crevices during oxidant release also mirror what was seen from other CR systems utilizing $\mathrm{KMnO}_{4}$ release [1] [27]. $\mathrm{KMnO}_{4}$ progresses initially from the preliminary contact between $\mathrm{KMnO}_{4}$ particles on the surface of the matrix and water (Figure 3(a)) to 24 hours (Figure $3(\mathrm{~b})$ ). The dissolution of the polymer bearing $\mathrm{KMnO}_{4}$ particles occurred as the surfaces were freely accessible to water in the batch reactors. The PCL polymer degraded slowly in the presence of aqueous solution, similar to what has been stated in the literature in that the embedded $\mathrm{KMnO}_{4}$ was released by dissolution-diffusion which resulted in the formation of $\mathrm{KMnO}_{4}$ solution that would be available to oxidize contaminants in the aqueous phase [1] [27] [29].

At the 24 hour mark, the SEM image indicates a maximum imbibition of the shell matrix (Figure 3(b)). It is at this point that the amount of hydrolysis in the PCL shell peaks, $\mathrm{KMnO}_{4}$ release decreased as the system transitioned from the 
dissolution phase to the diffusion phase followed by a controlled and slower release of $\mathrm{KMnO}_{4}$. Though beyond the sampling intervals for this study, a 1-week SEM picture was also taken of the system to show long-term effects of release on the polymer surface (Figure 3(c)). After a week of uninterrupted contact with water, $\mathrm{KMnO}_{4}$ dissolution left empty pores and larger crevices allowing the embedded $\mathrm{KMnO}_{4}$ to diffuse in aqueous solution, clearly seen in the SEM images and in agreement to what has been noted in the literature [29]. These results support the conjecture that the combined control CRBP delivery mechanism for PCL is more effective for long term release of the oxidant.

\subsection{Release Data}

The experimental release data for the $\mathrm{KMnO}_{4}$ from the PCL matrix (Figure 4) was found to best fit the following equation $\left(R^{2}=0.93\right)$ :

$$
Q_{t}=k t^{n}
$$

where $Q$ represents the mass fraction of $\mathrm{KMnO}_{4}$ released over time $t, k$ is a constant
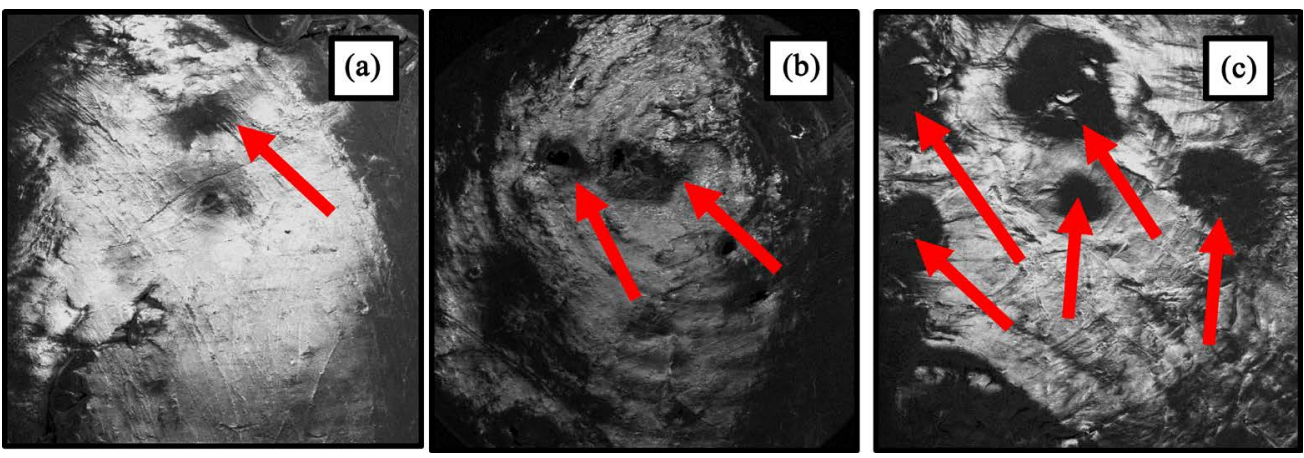

Figure 3. SEM images of the surface of encapsulated $20 \mathrm{wt} \% \mathrm{KMnO}_{4} \mathrm{CRBP}$ in (a) before release experiment, (b) after 1 day of release, (c) after 1 week of release. Magnification $=50 \mathrm{X}, \mathrm{kV}=5.00$ Scale bar $=500 \lambda \mathrm{m}$.

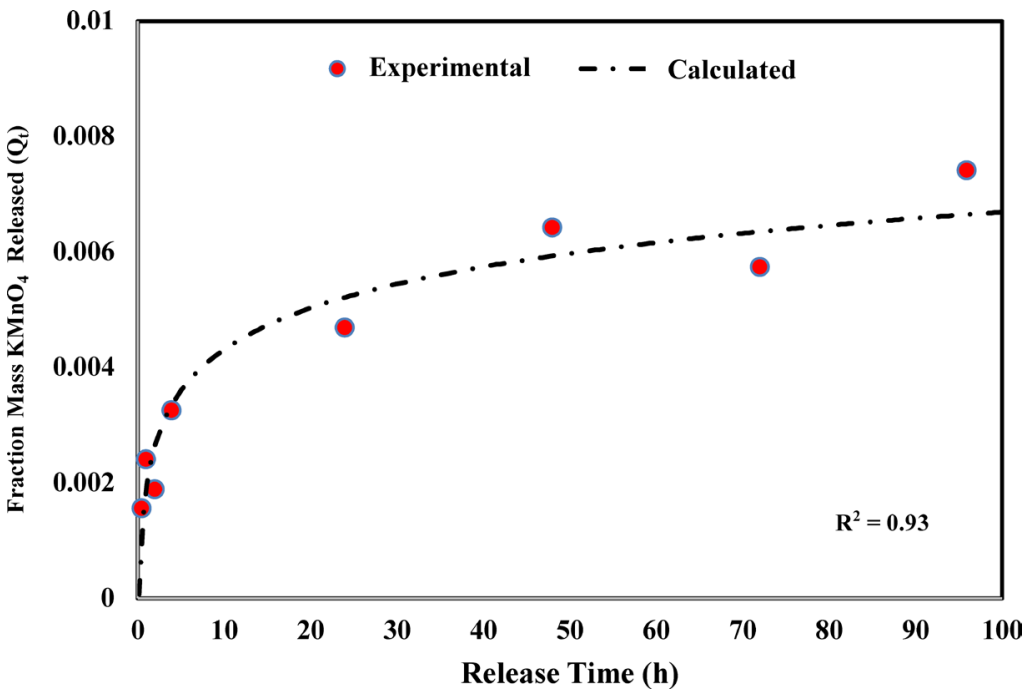

Figure 4. Experimental and calculated values (Equation (1)) for $\mathrm{KMnO}_{4}$ released over time from PCL in reagent grade water. 
incorporating polymer and oxidant characteristics, and $\mathrm{n}$ is the diffusional exponent. The fit of the data has been used to describe the release process of other oxidants from wax matrices as non-Fickian diffusion with constant pseudo-convection due to stress within the wax or polymer matrix itself [1] [27] [34]. These values of $\mathrm{n}$ and $\mathrm{k}$ can be gathered once Equation (1) has been linearized in logarithmic form (Equation (2)) and by plotting the experimental data to determine the slope and the intercept on the $\log Q_{t}$ axis, respectively.

$$
\log Q_{t}=\log k+n \log t
$$

Even with the linearized model, it has been shown that the estimated values might not necessarily simulate the nonlinear release kinetics [1] [34]. Therefore, estimation of parameters can be done with an optimization method that minimizes the sum of the square of errors (SSE) between the experimental and calculated data (Equation (3)).

$$
S S E=\sum_{t}\left(Q_{\exp }-Q_{\text {calc }}\right)^{2}
$$

\subsection{Comparison Release Studies}

The present study measured release of $\mathrm{KMnO}_{4}$ from PCL at a ratio of 1:5, otherwise known as $20 \mathrm{wt} \%$. Results from this present study were compared to parallel release studies with the same oxidant, $\mathrm{KMnO}_{4}$, encapsulated in different wax matrices at similar ratios. Kang et al. [1] examined the use of paraffin wax (Figure 5) to encapsulate the $\mathrm{KMnO}_{4}$ at a ratio of 1:5, similar to that of the present work.

In another study, Ighere and Chawla [27] used of Poly-methyl-methacrylate (PMMA) as polymer at a ratio of $1: 8 \mathrm{KMnO}_{4}$ to PMMA. PMMA is a hydrophobic synthetic resin and an ester of methacrylic acid generally produced from propylene (Figure 5). These comparable polymers had different chemical structures (Figure 5, Figure 6) than PCL which makes the information from their release compared to its release of $\mathrm{KMnO}_{4}$ valuable.

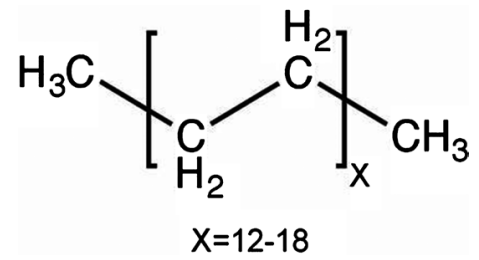

Figure 5. General structure of paraffin waxes.

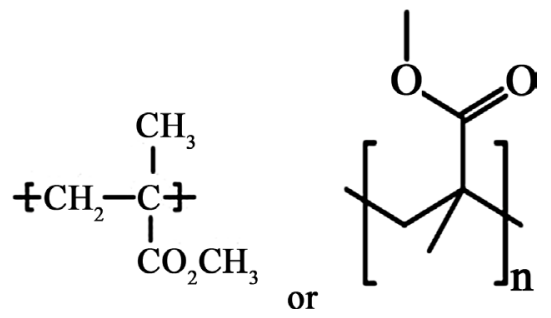

Figure 6. Structure of poly methyl methacrylate. 
Figure 7 shows the data in this present study with 1:5 ratio of $\mathrm{KMnO}_{4}$ to PCL agrees favorably with data from the compared studies which included Kang et al. [1] with 1:5 ratio $\mathrm{KMnO}_{4}$ to paraffin wax and that of Ighere and Chawla [27] with 1:8 ratio of $\mathrm{KMnO}_{4}$ to PMMA. The data from our present study was normalized by a factor of 20 , since its initial mass of oxidant in the PCL was $1 / 20^{\text {th }}$ of that utilized by the other comparable studies.

Furthermore, the PCL release data modeled also compare favorably with the model parameters from the comparative studies (Table 1). The values of $\mathrm{n}$ and $\mathrm{k}$ for all the studies were calculated from Equation 2 based on established research on this model by Sinclair and Peppas [34]. Evaluating these model parameters further indicated that the PCL release of $\mathrm{KMnO}_{4}$ of the present study aligned with this oxidant's release from the comparable studied polymers. The slight differences can be attributed to both the different chemical structures and physical characteristics of the different polymers used, as well as the higher mass amounts utilized in the data from the previous literature.

Though the present study paralleled the release from the compared studies, it most agreed with the Kang et al. [1] study. This was expected since that study and the present research utilized the same ratio of $1: 5$ or $20 \mathrm{wt} \%$. Further agreement

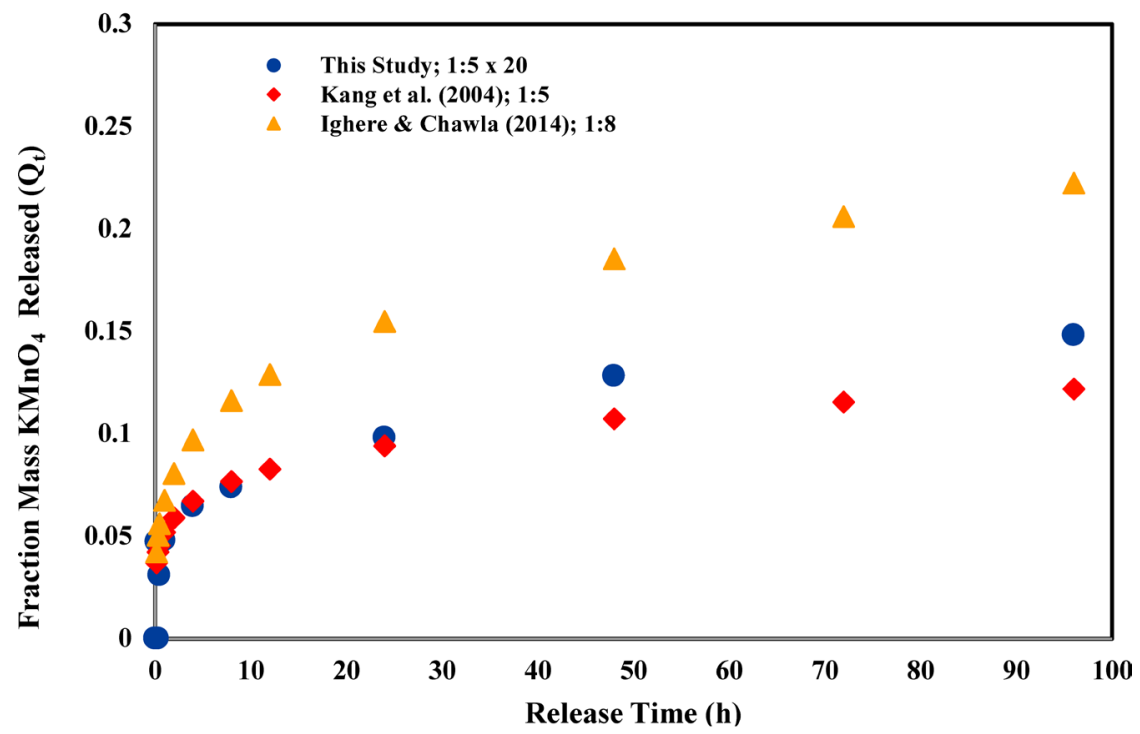

Figure 7. Theoretical data showing the rate of release of $\mathrm{KMnO}_{4}$ for this study alongside two other works utilizing $\mathrm{KMnO}_{4}$ as a controlled release oxidant in batch release experiments.

Table 1. Comparison of model parameters from experimental batch release.

\begin{tabular}{cccc}
\hline Study Polymer Type & KMnO $_{4}:$ Polymer Ratio & $\boldsymbol{n}$ & $\boldsymbol{k}$ \\
\hline $\begin{array}{c}\text { Present Study } \\
\text { Polycaprolactone (PCL) }\end{array}$ & $1: 5$ & 0.278 & 2.71 \\
$\begin{array}{c}\text { Kang et al. (2004) } \\
\text { Paraffin wax } \\
\begin{array}{c}\text { Ighere et al. (2014) } \\
\text { Polymethylmethacrylate (PMMA) }\end{array}\end{array}$ & $1: 5$ & 0.187 & 0.052 \\
\hline
\end{tabular}


between the present study and Kang et al. [1] study. This was noted in the calculation of SSE from Equation 3. The data of the present study was calculated to have a SSE value of $1.9 \times 10^{-5}$, while Kang et al. was $1.7 \times 10^{-4}$. This indicated that not only was the data relationships between these studies agreed, but also, that the data from the present data better fit the model as it was lower than that of the comparable study. Overall, the similarity of release data between the diversity of polymers shows that the CRBP with PCL provides effective release of the $\mathrm{KMnO}_{4}$ with the added benefit biodegradable nature of PCL, as mentioned previously [6] [21] [32].

\section{Conclusion}

Successful implementation of controlled release materials relies upon increased understanding of release patterns for CRBP systems. One such system includes Polycaprolactone polymer to control the slow release of $\mathrm{KMnO}_{4}$ oxidant as part of a patented process to remove contaminants from aqueous systems. Experiments were conducted to analyze the release data from this CRBP system into water at a ratio of 1:5 $\mathrm{KMnO}_{4}$ to PCL. Based on the experimental data, the fraction of the $\mathrm{KMnO}_{4}$ released from PCL into water was seen to continually increase over the 96 hour sampling period and resulted in the increase in size of cavities in the waxy surface of the polymer as seen in SEM images. Data from release of $\mathrm{KMnO}_{4}$ from PCL was found to fit non-Fickian diffusion after dissolution $\left(\mathrm{R}^{2}=0.93\right)$ similar to other systems that describe the dispersal of other oxidants from wax matrices. Release data and model parameters for data of this present study were also found to be comparable to previous release studies with the same oxidant, $\mathrm{KMnO}_{4}$, encapsulated in different wax matrices at similar ratios. Overall, the similarity of release data between the diversity of polymers shows that the CRBP with PCL provides effective release of the $\mathrm{KMnO}_{4}$ with the added benefit biodegradable nature of PCL.

\section{Acknowledgements}

The authors of this paper wish to thank the following for funding support: National Science Foundation (Award \#: 1740463); U.S. Department of Education GAANN Program (Award \#: P200A180074); U.S. Department of Education MSEIP Program: Program of Excellence in STEM (Award \#: P120A160115); Florida Agricultural \& Mechanical University, including the Title III Graduate Engineering Program for funding support; and North Carolina Agricultural \& Technical State University. Thanks are also given to the Civil and Environmental Engineering Department of the FAMU-FSU College of Engineering. The authors would like to thank, above all, their Lord and Savior Jesus the Christ for guidance during this endeavor.

\section{Conflicts of Interest}

The authors declare no conflicts of interest regarding the publication of this paper. 


\section{References}

[1] Kang, N., Hua, I., and Rao, P.S.C. (2004) Production and Characterization of Encapsulated Potassium Permanganate for Sustained Release as an in Situ Oxidant. Industrial \& Engineering Chemistry Research, 43, 5187-5193. https://doi.org/10.1021/ie0499097

[2] Ross, C., Murdoch, L.C., Freedman, D.L. and Siegrist, R.L. (2005) Characteristics of Potassium Permanganate Encapsulated in Polymer. Journal of Environmental Engineering, 131, 1203-1211. https://doi.org/10.1061/(ASCE)0733-9372(2005)131:8(1203)

[3] Lee, B.S., Kim, J.H., Lee, K.C., Kim, Y.B., Schwartz, F.W., Lee, E.S., Woo, N.C. and Lee, M.K. (2009) Efficacy of Controlled-Release $\mathrm{KMnO}_{4}$ (CRP) for Controlling Dissolved TCE Plume in Groundwater: A Large Flow-Tank Study. Chemosphere, 74, 745-750. https://doi.org/10.1016/j.chemosphere.2008.10.062

[4] Harris, M.S. (1981) Preparation and Release Characteristics of Potassium Chloride Microcapsules. Journal of Pharmaceutical Sciences, 70, 391-394.

https://doi.org/10.1002/jps.2600700413

[5] Nelson, M.D., Parker, B.L., Al, T.A., Cherry, J.A. and Loomer, D. (2001) Geochemical Reactions Resulting from in Situ Oxidation of PCE-DNAPL by $\mathrm{KMnO}_{4}$ in a Sandy Aquifer. Environmental Science \& Technology, 35, 1266-1275. https://doi.org/10.1021/es001207v

[6] Luster-Teasley, S., Onochie, P., and Shirley, V. (2010) Encapsulation of Potassium Permanganate Oxidant in Biodegradable Polymers to Develop a Novel Form of Controlled-Release Remediation. In: Shah, V., Ed., Emerging Environmental Technologies, Vol. 2, Springer, Dordrecht, 39-55.

https://doi.org/10.1007/978-90-481-3352-9 2

[7] Ma, Y., Feng, Y., Feng, Y., Liao, G., Sun, Y., and Ma, J. (2020) Characteristics and Mechanisms of Controlled-Release $\mathrm{Kmno}_{4}$ for Groundwater Remediation: Experimental and Modeling Investigations. Water Research, 171, Article ID: 115385.

https://doi.org/10.1016/j.watres.2019.115385

[8] Cherry, A.K. (1962) Use of Potassium Permanganate in Water Treatment. Journal of American Water Works Association, 54, 417-424. https://doi.org/10.1002/j.1551-8833.1962.tb00862.x

[9] O'Connor, D., Hou, D., Ok, Y.S., Song, Y., Sarmah, A.K., Li, X. and Tack, F.M.G. (2018) Sustainable in Situ Remediation of Recalcitrant Organic Pollutants in Groundwater with Controlled Release Materials: A Review. Journal of Controlled Release, 283, 200-213. https://doi.org/10.1016/j.jconrel.2018.06.007

[10] Schwartz, F.W. (2005) Semi-Passive, Chemical Oxidation Schemes for the Long-Term Treatment of Contaminants. https://doi.org/10.2172/885059 http://www.osti.gov/scitech/servlets/purl/861451

[11] Lee, E.S., and Schwartz, F.W. (2007) Characteristics and Applications of Controlled-Release $\mathrm{KMnO}_{4}$ for Groundwater Remediation. Chemosphere, 66, 2058-2066. https://doi.org/10.1016/j.chemosphere.2006.09.093

[12] Yuan, B.L., Chen, Y.M., and Fu, M.-L. (2012) Degradation Efficiencies and Mechanisms of Trichloroethylene (TCE) by Controlled-Release Permanganate (CRP) Oxidation. Chemical Engineering Journal, 192, 276-283.

https://doi.org/10.1016/j.cej.2012.03.049

[13] Liang, S. H., Chen, K. F., Wu, C. S., Lin, Y. H., and Kao, C. M. (2014) Development of $\mathrm{KMnO}_{4}$-Releasing Composites for in Situ Chemical Oxidation of TCE-Contaminated Groundwater. Water Research, 54, 149-158. 
https://doi.org/10.1016/j.watres.2014.01.068

[14] Woodruff, M.A., and Hutmacher, D.W. (2010) The Return of a Forgotten Polymer-Polycaprolactone in the 21st Century. Progress in Polymer Science, 35, 1217-1256. https://doi.org/10.1016/j.progpolymsci.2010.04.002

[15] Brewster, M.L., Annan, A.P., Greenhouse, J.P., Kueper, B.H., Olhoeft, G.R., Redman, J.D., and Sander, K.A. (1995) Observed Migration of a Controlled DNAPL Release by Geophysical Methods. Groundwater, 33, 977-987. https://doi.org/10.1111/j.1745-6584.1995.tb00043.x

[16] Clark II, C.J., Rao, P.S.C. and Annable, M.D. (2003) Degradation of Perchloroethylene in Cosolvent Solutions by Zero-Valent Iron. Journal of Hazardous Materials, 96, 65-78. https://doi.org/10.1016/S0304-3894(02)00162-0

[17] Clark II, C. J., Chen, X., and Babu, S. (2005) Degradation of Toxaphene by Zero-Valent Iron and Bimetallic Substrates. Journal of Environmental Engineering, 131, 1733-1739. https://doi.org/10.1061/(ASCE)0733-9372(2005)131:12(1733)

[18] Shih, Y.-J., Binh, N. T., Chen, C.-W., Chen, C.-F., and Dong, C.-D. (2016) Treatability Assessment of Polycyclic Aromatic Hydrocarbons Contaminated Marine Sediments Using Permanganate, Persulfate and Fenton Oxidation Processes. Chemosphere, 150, 294-303. https://doi.org/10.1016/j.chemosphere.2016.01.112

[19] Hu, Y. B., Zhang, M. Y., Qiu, R. L., and Li, X. Y. (2018) Encapsulating Nanoscale Zero-Valent Iron with a Soluble $\mathrm{MgOH}_{2}$ Shell for Improved Mobility and Controlled Reactivity Release. Journal of Materials Chemistry A, 6, 2517-2526. https://doi.org/10.1039/C7TA08605A

[20] Yan, Y. E., and Schwartz, F. W. (2000) Kinetics and Mechanisms for TCE Oxidation by Permanganate. Environmental Science \& Technology, 34, 2535-2541. https://doi.org/10.1021/es991279q

[21] Luster-Teasley, S., Jackson, C., and Rogers, C. (2011) Inactivation of Pathogens in Agricultural Wastewater Using Controlled Release Chemical Oxidation Polymer System. Proceedings of the 2011 World Environmental and Water Resources Congress, Palm Springs, 22-26 May 2011, 1562-1571. https://doi.org/10.1061/41173(414)163

[22] Shaabani, A., Tavasoli-Rad, F. and Lee, D.G. (2005) Potassium Permanganate Oxidation of Organic Compounds. Synthetic Communications, 35, 571-580. https://doi.org/10.1081/SCC-200049792

[23] Kao, C.M., Huang, K.D., Wang, J.Y., Chen, T.Y. and Chien, H.Y. (2008) Application of Potassium Permanganate as an Oxidant for in Situ Oxidation of Trichloroethylene-Contaminated Groundwater: A Laboratory and Kinetics Study. Journal of Hazardous Materials, 153, 919-927. https://doi.org/10.1016/j.jhazmat.2007.09.116

[24] Luster-Teasley, S., Price, D., and Worku, D. (2009) Encapsulation of Potassium Permanganate Oxidant in Polymers. 2007 National Conference on Environmental Science and Technology, New York, 12-14 September 2009, 279-284. https://doi.org/10.1007/978-0-387-88483-7 38

[25] Woo, N.C., Hyun, S.G., Park, W.W., Lee, E.S. and Schwartz, F.W. (2009) Characteristics of Permanganate Oxidation of TCE at Low Reagent Concentrations. Environmental Technology, 30, 1337-1342. https://doi.org/10.1080/09593330902840342

[26] Loomer, D.B., Al, T.A., Banks, V.J., Parker, B.L. and Mayer, K.U. (2011) Manganese and Trace-Metal Mobility under Reducing Conditions Following in Situ Oxidation of TCE by $\mathrm{KMnO}_{4}$ : A Laboratory Column Experiment. Journal of Contaminant Hydrology, 119, 13-24. https://doi.org/10.1016/j.jconhyd.2010.08.005 
[27] Ighere, J. O., and Chawla, R. C. (2014) Controlled-Release Analysis of Potassium Permanganate Using Pmma Matrix. Journal of Minerals and Materials Characterization and Engineering, 2, 539-544. http://dx.doi.org/10.4236/jmmce.2014.26055

[28] Dong, S. S., Wang, J., Wang, Y. L., Zhang, J. B., Zhou, D. D., \& Shao, C. Y. (2013) Preparation and Releasing Characteristics of Controlled Release Potassium Permanganate. Materials Science Forum, 743-744, 782-788.

https://doi.org/10.4028/www.scientific.net/MSF.743-744.782

[29] Xiong, H., Huo, M., Zhou, D., Dong, S., and Zou, D. (2016) Characteristics and Kinetics Simulation of Controlled-Release $\mathrm{KMnO}_{4}$ for Phenol Remediation. Water Science and Technology, 74, 647-654. https://doi.org/10.2166/wst.2016.233

[30] Johnson, R.M., Mwaikambo, L.Y. and Tucker, N. (2003) Biopolymers. Rapra Technology, Shrewsbury.

[31] Yuan, B., Li, F., Chen, Y., and Fu, M.-L. (2013) Laboratory-Scale Column Study for Remediation of TCE-Contaminated Aquifers Using Three-Section Controlled-Release Potassium Permanganate Barriers. Journal of Environmental Sciences, 25, 971-977. https://doi.org/10.1016/S1001-0742(12)60134-X

[32] Luster-Teasley, S. (2013) Controlled Release Remediation System and Composition. US Patent No. 8519061B2.

[33] Quaglia, F., Barbato, F., De Rosa, G., Granata, E., Miro, A. and La Rotonda, M.I. (2001) Reduction of the Environmental Impact of Pesticides: Waxy Microspheres Encapsulating the Insecticide Carbaryl. Journal of Agricultural and Food Chemistry, 49, 4808-4812. https://doi.org/10.1021/jf010418w

[34] Sinclair, G. W. and Peppas, N. A. (1984) Analysis of Non-Fickian Transport in Polymers Using Simplified Exponential Expressions. Journal of Membrane Science, 17, 329-331. https://doi.org/10.1016/S0376-7388(00)83223-8 